\title{
HYPERSONIC BOUNDARY LAYER STABILITY OVER A FLARED CONE IN A QUIET TUNNEL
}

\author{
Jason T. Lachowicz* and Ndaona Chokani ${ }^{\dagger}$ \\ North Carolina State University, Raleigh NC 27695 \\ and \\ Stephen P. Wilkinson ${ }^{\ddagger}$ \\ NASA Langley Research Center, Hampton VA 23681
}

\begin{abstract}
$\underline{\text { Abstract }}$
Hypersonic boundary layer measurements were conducted over a flared cone in a quiet wind tunnel. The flared cone was tested at a freestream unit Reynolds number of $2.82 \times 10^{6} / \mathrm{ft}$ in a Mach 6 flow. This Reynolds number provided laminar-to-transitional flow over the model in a low-disturbance environment. Point measurements with a single hot wire using a novel constant voltage anemometry system were used to measure the boundary layer disturbances. Surface temperature and schlieren measurements were also conducted to characterize the laminar-to-transitional state of the boundary layer and to identify instability modes. Results suggest that the second mode disturbances were the most unstable and scaled with the boundary layer thickness. The integrated growth rates of the second mode compared well with linear stability theory in the linear stability regime. The second mode is responsible for transition onset despite the existence of a second mode sub-harmonic. The sub-harmonic wavelength also scales with the boundary layer thickness. Furthermore, the existence of higher harmonics of the fundamental suggests that non-linear disturbances are not associated with "high" free stream disturbance levels.
\end{abstract}

\section{Nomenclature}

A Disturbance rms amplitude (square of power spectral density, arbitrary units).

f Frequency $(\mathrm{kHz})$

$\mathrm{R} \quad\left(\operatorname{Re}_{\mathrm{S}}\right)^{1 / 2}$

$\mathrm{Re}_{\mathrm{S}}$ Reynolds number based upon freestream conditions and surface distance from the apex of the cone, $\mathrm{S}$

$\mathrm{r}_{\mathrm{b}} \quad$ Base radius of cone

$\mathrm{S} \quad$ Distance along the surface of the model, measured from the apex of the cone model

To Total temperature

$\mathrm{T}_{\mathrm{w}} \quad$ Surface static temperature

$\rho U$ Mass flux

Vs Constant voltage anemometer output voltage

\footnotetext{
*Graduate Research Assistant, Department of Mechanical and Aerospace Engineering, Student Member AIAA. Presently, NRC Research Associate, NASA Langley.

$\dagger$ Associate Professor, Department of Mechanical and Aerospace Engineering, Member AIAA.

¥Group Leader, Quiet Tunnel \& Transition Group, Flow Modeling and Control Branch, Fluid Mechanics and Acoustics Division, Senior Member AIAA.
}

$\mathrm{X}$ Coordinate along the cone model axis of symmetry, measured from the apex of the cone

$\mathrm{Y}$ Coordinate perpendicular to the cone axis of symmetry, measured from the cone axis of symmetry

$\mathrm{Y}_{\mathrm{w}} \quad \mathrm{Y}$-location of the wall surface

Greek

$-\alpha_{i} \quad$ Non-dimensional amplification rate, $-\alpha_{i}=\frac{1}{2 \mathrm{~A}} \frac{\mathrm{dA}}{\mathrm{dR}}$

$\delta \quad$ Boundary layer thickness

$\lambda \quad$ Disturbance wavelength

$\eta \quad$ Non-dimensional Y-distance, $\eta=\frac{\left(Y_{-}-Y_{W}\right) R}{S}$

Subscripts

$\infty \quad$ Conditions in freestream

rms Root mean square of fluctuating component

Superscripts

' Fluctuating component of a time dependent quantity

( ) Time average of a particular quantity

Introduction

The change from laminar to turbulent flow in the hypersonic boundary layer is accompanied by large changes in both heat transfer and skin-friction drag. These changes are important to the aerodynamic design of hypersonic vehicles since the aerodynamic coefficients are very sensitive to the large changes in heat transfer and skin-friction that accompany transition. ${ }^{1}$ Furthermore, the stability, control, and structural design of the vehicle are affected due to the increased thermal and aerodynamic loading.

The conical geometry is prevalent in many hypersonic aerodynamic applications, but only a few stability experiments $^{2}$ of hypersonic cone boundary layers have been conducted. These studies have provided a fundamental understanding of the hypersonic boundary layer stability problem. However, these few stability experiments have been conducted in conventional hypersonic wind tunnels where relatively large freestream disturbances occur. The primary source of the free stream disturbances is acoustic radiation from convecting eddies generated by the turbulent boundary layer on the nozzle wall. 3,4 The frequency content of this incident noise field provides a stimulus to excite disturbances in the hypersonic boundary layer which may lead to transition. Thus, some of the observed anomalies between experiment and theory ${ }^{5,6}$ may be due to effects of the wind tunnel noise.

This paper is declared a work of the US Government and is not subject to copyright protection in the United States. 
In order to provide a more reliable test environment for the experimentalist, the NASA Langley Research Center has developed a series of supersonic/hypersonic quiet tunnels. ${ }^{7}$ In these facilities, the free stream noise is maintained at low levels by treating the settling chamber flow and maintaining the nozzle wall boundary layer in a laminar state. Hence, transition on the nozzle wall is delayed providing lower free stream disturbance levels in the quiet tunnel relative to conventional tunnels.

The primary objective of the present study is to obtain experimental hypersonic boundary layer stability data over a conical body in a quiet tunnel. In a parallel paper, Ref. 8, the disturbance environment of the quiet tunnel used in the present work is documented. In this paper, the first hypersonic boundary layer stability measurements obtained in a quiet tunnel are presented.

\section{Experimental Apparatus}

\section{$\underline{\text { Test Facility }}$}

All tests were conducted in the NASA Langley Nozzle Test Chamber Facility. This is an open-jet blow-down facility, and was equipped for the present tests with a slow-expansion, axisymmetric, quiet Mach 6 nozzle. The nozzle, which is more fully described in Ref. 9, had a throat diameter of 1.00", exit diameter of 7.49", and length from throat to exit of 39.76". The nozzle is equipped with an annular throat slot to remove the boundary layer that develops upstream of the throat. As a result, the boundary layer that forms on the nozzle wall remains in a laminar state until far downstream. This provides low disturbance levels in the nozzle test section. The nozzle may be operated over a range of stagnation pressures from 80 to 200 psia, and stagnation temperatures up to $400^{\circ} \mathrm{F}$. Run times from minutes to several hours are possible.

\section{Test Model}

The model, used in this study, was a 20" long cone with a curved-flare afterbody, shown in Fig. 1. For sake of brevity, this model is referred to as the flared cone. The straight cone surface extended from $X=0$ " to $X=10$ ", with a semivertex angle of $5^{\circ}$. The flare surface had a radius of curvature of 93.07", and extended from $X=10^{\prime \prime}$ to $X=20 "$ ". The sharp model tip had a nominal radius of $0.0001 "$. The model surface was instrumented with 29 pressure orifices and 51 thermocouple gages placed along diametrically opposite rays as shown in the side view of Fig. 1. Hot-wire boundary layer surveys were conducted along a ray located $90^{\circ}$ from the surface measurement rays as shown in the top view of Fig. 1.

The flared cone was used instead of a straight cone in order to induce transition on the model within the quiet flow capability of the tunnel. The model surface was of high fidelity, and had a maximum rms radius error of less that $2.8 \%$ of the model boundary layer thickness.

\section{Hot-Wire Probes}

The hot-wire probes were constructed of $10 \%$ platinum plated tungsten wire of $100 \mu \mathrm{in}$. diameter. The wire was soldered onto $0.005 "$ diameter stainless steel broaches which were attached to the main probe body. The nominal length-to-diameter ratio of the wire was 210 . The wire was slack to minimize the "strain-gage effect". An electrical contact probe was located about $0.005 "$ below the broaches in order to determine the location of the model surface.

\section{Hot-Wire Anemometer}

The hot-wire anemometer system used in the present work was a new, proprietary constant voltage anemometer (CVA). In this system, a steady DC voltage was maintained across the hot wire through the use of a composite-amplifier-compensation circuit. The operating principles of the CVA are described in detail in Ref. 11. Only the CVA, in contrast to attempts with constant current and constant temperature anemometers, provided the ability to obtain measurable signals in the freestream of the quiet nozzle flow. The reason for the better performance of the CVA has not yet been investigated. The present CVA system had a bandwidth of about 350 $\mathrm{kHz}$ with a $40 \mathrm{~dB} /$ decade roll-off.

The CVA operation was computer controlled for all tests conducted. At each boundary layer measurement point, the wire voltage was automatically changed through 7 levels; the voltage magnitudes were optimized for the individual wires used. The constant wire voltage was monitored throughout testing using a $5 \frac{1 / 2}{2}$ digit digital multimeter (DMM). The DMM was also used to measure the mean CVA output signal. The rms and fluctuating components were measured from the AC-coupled CVA output signal. An analog true RMS voltmeter was used to determine the CVA rms output voltage. Prior to measurement, the CVA output was high-pass filtered at 1 $\mathrm{kHz}$ and low-pass filtered at $1 \mathrm{MHz}$. An 8-Bit digital oscilloscope was used to obtain time traces of the fluctuating CVA output voltage. The high- and low-pass filter settings were $1 \mathrm{kHz}$ and $630 \mathrm{kHz}$, respectively, and the sampling rate was $2 \mathrm{MHz}$. Standard FFT procedures employing a Hanning window, data length of 512 points, and 78 averages were used to obtain the spectra.

A calibration procedure for the CVA was developed, and is outlined in Ref. 12. This procedure enabled mean and rms mass flux and total temperatures in the boundary layer to be obtained. The comparisons of the present data with computational predictions verified the validity of the procedure for mean flow quantities. However, the accuracy of the quantitative rms data could not be verified for the fixed-time-compensation CVA system. Nevertheless, the absence of quantitative fluctuation data did not prevent an analysis of the spatial amplification rates in the linear and weakly non-linear stability regimes. In the linear stability region, fluctuations are expected to grow (or decay) exponentially. This exponential growth is described by normal mode decomposition ${ }^{5}$ which specifies that the amplification rate is the same for separate fluctuation components (pressure, velocity, etc.). Thus, an uncalibrated approach is valid in the linear stability region as verified from both controlled ${ }^{13}$ and uncontrolled ${ }^{6}$ stability experiments. In the weakly non-linear stability regime, analysis of the present data indicated that the experimentally-derived amplification rates are mainly of a mass-flux nature ${ }^{12}$.

Experimental Approach

$\underline{\text { Test Conditions }}$ 
The stagnation conditions for the present tests were a temperature of $810^{\circ} \mathrm{R}$ and a pressure of $130 \mathrm{psia}$. The measured freestream Mach number was 5.91. These conditions yielded a freestream unit Reynolds number of $2.82 \times 10^{6} / \mathrm{ft}$

Prior to the present tests, the characteristics of the freestream flow were documented through a series of pitot-probe and hot-wire measurements. The details of the freestream measurements are presented in Refs. 8 \& 12, but a few salient features are presented here. The pitotprobe surveys verified that the nozzle mean flow was uniform within the flow test volume. Within this volume the Mach number was $5.91 \pm 0.08$. Measurements in cross-sectional planes indicated that the mean flow was axisymmetric and varied by no more than $1 \%$ in the test volume which encompassed the model. The RMS data showed that the low-level disturbance field was axisymmetric and was associated with sound-mode generation of the nozzle wall turbulent boundary layer.

Measurements

The measurements were conducted in two stages. First, model surface temperature and schlieren imaging measurements were conducted, and then hot-wire boundary layer surveys were conducted. The surface temperature measurements were used to verify that the model was in thermal equilibrium, and to estimate the location of transition onset. The schlieren images were used to verify the laminar-to-transitional state of the boundary layer, and to identify the character of the instability modes.

The hot wire boundary layer surveys were conducted at 17 streamwise stations, spaced $0.5^{\prime \prime}$ apart over the range $X=10.97 "(R=1610)$ to $X=18.97 "(R=2120)$. At each streamwise station, the wire was traversed perpendicular to the cone axis of symmetry. The mean and rms measurements were obtained at 13 points clustered near the boundary layer edge. At each measurement point, 7 wire voltages were applied. The rms profiles were then inspected to determine the maximum energy (rms) location. Wave traces were subsequently measured at the maximum energy locations using the largest, practicable wire voltage. At this operating condition, the CVA is more sensitive to changes in the mass flux as opposed to changes in the total temperature ${ }^{12}$. In addition, the CVA signal-to-noise ratio is improved.

\section{$\underline{\text { Results }}$ \\ Surface Temperature Data}

Fig. 2 presents the experimental and computational surface temperatures. The temperatures are shown along the left ordinate and the flared-cone surface coordinates along the right ordinate. The experimental surface temperature error is $\pm 2^{\circ} \mathrm{R} \quad\left( \pm 0.0025 \mathrm{To}_{\infty}\right)$. The computational values ${ }^{14}$ represent laminar adiabatic wall temperatures. Over the range, $\mathrm{R}=690-1700$, the flow is laminar and the experimental data compare well with the computational data. Further downstream, $\mathrm{R}=1800-2110$, there is sharp temperature rise region. This rise is associated with transition since a transitional boundary layer is heated and some of this heat is convected to the model surface via turbulent-like vortices. An estimate of transition onset was determined as the intersection point of two straight lines passing through the laminar region and sharp temperature rise region. Based on this criterion, the transition onset is estimated to be in the range $\mathrm{R}=1960$ 1990. The estimate compares well with linear stability theory ${ }^{9}$ which predicts an $\mathrm{N}$-factor for the most unstable frequency of about 8 at $\mathrm{R}=1975$. Downstream of $\mathrm{R}=2110$, the temperature decreases due to the combined effect of a relatively cold model base and, possibly, the flow field tending to fully turbulent flow.

\section{Schlieren Data}

Schlieren data are presented in Fig. 3. These measurements were conducted over the aft 3.5 " of the model which was positioned downstream of the nozzle exit plane. A wavy structure can be identified near the edge of the boundary layer. The wavelength of these waves is measured to be approximately twice the boundary layer thickness. These waves occur in wave packets and are associated with second mode disturbances ${ }^{2,6}$. The second mode disturbances are first detected at about $\mathrm{R}=2025$ according to a closer examination of the video records used to construct Fig. 3. This location is slightly downstream of transition onset as estimated from the surface temperature measurements.

\section{Boundary Layer Mean Data}

The experimental and computational boundary layer thickness distributions are presented in Fig. 4. Note that the computational ${ }^{14}$ boundary layer thickness distribution was curve fit using a second order polynomial, and the experimental error is $\pm 2 \%$ of the plotted values. Except for a few locations over the range, $\mathrm{R}=1610-1915$, the experimental $\delta$ is slightly lower than the computational $\delta$. As discussed below this suggests a misalignment of the model such that the boundary layer measurement ray is on the windward side of the model. From $R=1945$ to $R=2120$, the experimental $\delta$ becomes greater than the computational $\delta$, confirming the transitional nature of the boundary layer over this region. For $1610 \leq \mathrm{R} \leq 1915$, the close agreement between the laminar flow computational predictions and the experimental data suggests that, experimentally, the mean flow is laminar over this region (i.e. no mean flow distortion). This laminar character is seen more clearly with the aid of Figs. 5 and 6 which are discussed next.

The experimental mean total temperature and mass flux profiles are presented in Figs. 5 and 6 at 4 streamwise locations. Also, laminar total temperature and mass flux profiles, computed from the Navier-Stokes code of Ref. 14 , are presented as the solid lines in Figs. 5-6. At $\mathrm{R}=1785$, the experimental and computational data compare well; no effect of model misalignment is evident. The good agreement with computational data at $\mathrm{R}=1785$ is typical of all total temperature data over the range, $1610 \leq$ $\mathrm{R} \leq 1915$. This is consistent with the boundary layer thickness, confirming the laminar flow region, $\mathrm{R} \leq 1915$. At $\mathrm{R}=1945$, the transitional nature of the boundary layer becomes evident due to the slight total temperature distortion from $\eta=5.27(0.706 \delta)$ to $\eta=6.62(0.887 \delta)$ and mass flux distortion from $\eta=5.61(0.751 \delta)$ to $\eta=6.93$ 
$(0.928 \delta)$. These distortions become more pronounced further downstream. At $\mathrm{R}=2035$, the total temperature is distorted from about $\eta=5.09(0.734 \delta)$ to $\eta=6.39(0.921 \delta)$. At the most downstream location, $\mathrm{R}=2120$, the lower portion of the boundary layer region is distorted from $\eta=4.49(0.680 \delta)$ to $\eta=6.18(0.935 \delta)$, marking a "high fluctuating disturbance" region. Overall, the mean flow is distorted in the range, $(0.71-0.93) \delta$, which is in the vicinity of the critical layer.

The uncalibrated rms profiles are presented in Fig. 7. The hashed region, from $\mathrm{R}=1610$ to $\mathrm{R}=1750$ represents the measurement range over which the rms signal-to-noise $(\mathrm{S} / \mathrm{N})$ ratio was $\leq 1$. Thus, the data at these five streamwise locations were not considered further in this study. Also shown in Fig. 7 is the locus of the maximum disturbance energy. The position of the maxima are at about 80 to $90 \%$ of the boundary layer thickness which is in good agreement with the eigenfunction maxima locations predicted by stability theory. For the range, $1785 \leq \mathrm{R} \leq$ 1945 , the $\mathrm{S} / \mathrm{N}>1$, but no clear indication of rapid maximum rms amplitude growth is evident. However, just downstream, a rapid growth region occurs over the range, $1975 \leq \mathrm{R} \leq 2120$. The location of $\mathrm{R}=1975$, is in good agreement with the transition onset location estimated from the surface temperature data.

The CVA calibration of Ref. 12 was used to convert the data of Fig. 7 into rms mass flux and total temperatures. These data are normalized by the mean mass flux and total temperatures. For brevity, the normalized rms quantities are termed rms fluctuations in the presentation of Figs. 89. (Note that the instrumentation noise was not subtracted for these data and thus only regions where the $\mathrm{S} / \mathrm{N}>1$ represent the true fluctuation levels).

The mass flux and total temperature rms fluctuations are presented as a function of $\mathrm{R}$ in Fig. 8. The rms fluctuations are presented at the maximum energy locations. At the most upstream location, $\mathrm{R}=1785$, the mass flux and total temperature rms fluctuations are $2.1 \%$ and $0.5 \%$, respectively. Over the region, $1785<\mathrm{R} \leq 1945$, the mass flux and total temperature rms fluctuations increase only slightly from their upstream values. However, over this region, $\mathrm{S} / \mathrm{N} \approx 1$, and thus the actual mass flux and total temperature rms fluctuations are lower than the values shown. Further downstream, $\mathrm{S} / \mathrm{N}>1$, and the fluctuation levels increase for both flow variables. At the most downstream location, $\mathrm{R}=2120$, the rms fluctuations reach a maximum of $11 \%$ and $2.3 \%$ for the mass flux and total temperature, respectively. Thus, from $\mathrm{R}=1975$ to $\mathrm{R}=2120$, the mass flux rms fluctuation increases by a factor of 5.2, comparable to the total temperature increase of 4.6. However, considering each location over the full R-range, the mass flux rms fluctuation is a factor of 4-8.5 larger than the total temperature rms fluctuation. Consequently, the second mode disturbances are predominantly of a mass flux, or acoustic, nature.

A typical set of boundary layer mass flux and total temperature rms fluctuation profiles are presented in Fig. 9 at $\mathrm{R}=2035$. This location is in the rapid disturbance growth region. For both the mass flux and total temperature, the rms fluctuation maxima occur at $\eta=6.17$, or $0.889 \delta$. Thus, the rms maxima occur in the critical layer region, $(0.8-0.9) \delta$, as expected. In addition, the calibrated maxima compare within $1 \%$ of the uncalibrated maxima. Overall, for the entire streamwise range surveyed, the uncalibrated maxima were within $0-3 \%$ of the calibrated maxima.

\section{Boundary Layer Fluctuation Data}

The fluctuation spectra are presented in Figs. 10 and 11 at the maximum energy locations. Fig. 11 represents the frontal view of the fluctuation spectra of Fig. 10. Prior to discussing the specific instability waves of interest from this data, the second mode fluctuations $(\mathrm{f}=210-290 \mathrm{kHz})$ are first discussed.

As shown in Fig. 10, for $\mathrm{R} \geq 1975$, the second mode amplitudes increase in the streamwise direction. Furthermore, the frequency of the most amplified second mode disturbances increases in the streamwise direction as observed from Fig. 11. This observation verifies the boundary layer tuning of the disturbances and also confirms their second mode character ${ }^{6}$. Specifically, over the range, $1975 \leq \mathrm{R} \leq 2060$, the frequency of the second mode most amplified disturbances increases, corresponding to the boundary layer thickness decrease over this same range as observed in Fig. 4. Over the range, $2060<\mathrm{R} \leq 2120$ (last 3 streamwise locations), the second mode most amplified disturbance frequency remains constant at $254 \mathrm{kHz}$, suggesting a reduction in disturbance growth rate over this range. This is consistent with the "small" change in boundary layer thickness over this same range as observed from Fig. 4. Overall, the boundary layer tuning of the disturbances is consistent with the boundary layer thickness data of Fig. 4.

Although the second mode disturbance growth rate decreases over the last 3 streamwise locations, the amplitudes grow to the last measurement station of $\mathrm{R}=2120$ as observed from Fig. 10. Since Kimmel ${ }^{6,15}$ defines transition onset over a straight cone as the streamwise location where the second mode amplitudes reach a maximum before decaying, transition onset does not occur in the present work. However, transition onset estimated from the surface temperatures occurs in the range $\mathrm{R}=1960-1990$ as previously discussed. Thus, the transition onset location as defined by Kimmel may not work well for a flared-cone configuration due to the rapid disturbance growth relative to the straight cone. (Kimmel's definition seems more appropriate for defining the end of transition as opposed to the beginning). In summary, transition onset occurs in the vicinity of second mode rapid disturbance growth, $\mathrm{R}=1975$.

For $1945 \leq \mathrm{R} \leq 2120$ the most unstable frequencies in terms of the maximum $\mathrm{N}$-factor is in the frequency range, 245-255 kHz. Based on $\mathrm{LST}^{10}$, the most unstable frequency range over the same R-range is $220-230 \mathrm{kHz}$. Subsequent measurements presented in the next section clearly indicate a most unstable frequency range of 218$228 \mathrm{kHz}$, confirming that the frequency shift in the present data is caused by model misalignment. This frequency shift is attributed to a corresponding change in boundary layer thickness. 
The fluctuation spectra through the boundary layer at a fixed streamwise location, $\mathrm{R}=2120$, is presented in Fig. 12. (These data were measured using a different hot-wire than all other data presented in this paper.) For $\mathrm{R}=2120$, the second mode frequency band, $210-290 \mathrm{kHz}$, is constant throughout the boundary layer as expected. In addition, the amplitude profile of the second mode band, with respect to distance from the wall, is similar to the stability theory eigenfunction profile. The second mode amplitudes approach zero at both the wall and boundary layer edge which is consistent with the prescribed boundary conditions of a subsonic, second mode. ${ }^{16}$

The amplification rates in 3 frequency bands of interest are discussed in Figs. 13-22. The first frequency band of interest is from $65<\mathrm{f}<85 \mathrm{kHz}$. This frequency band is associated with first mode disturbances but these disturbance amplitudes are not clearly discernible in Fig. 10. The second band of interest, $110<\mathrm{f}<130 \mathrm{kHz}$, is associated with a sub-harmonic of the second mode. This band is discernible in Fig. 10 over the last three Rlocations. As previously discussed, the last band of interest, $210<\mathrm{f}<290 \mathrm{kHz}$, is associated with the second mode and is clearly discernible in Fig. 10. (Note that an additional frequency band of interest, $0<\mathrm{f}<80 \mathrm{kHz}$, is not associated with Görtler vortices generated by the curved flare since subsequent measurements over a straight cone showed similar low-frequency growth. These low-frequency disturbances may represent the footprint of the freestream spectra. The growth of the low-frequencies are consistent with the growth of the freestream disturbances which would include the influence of the Görtler mode on the nozzle wall.)

Figs. 13-19 present the spectra of the amplification rates, fluctuation amplitudes, and instrumentation/CVA noise amplitudes at 7 streamwise (R) locations on the model flare. (Note that the right ordinate amplitude scale varies from Figs. 13 to 19. In addition, the ratio of fluctuation amplitudes to noise amplitudes in the spectra are used to estimate the $\mathrm{S} / \mathrm{N}$ ratio.) The amplification rate data of Figs. 13-19 are discussed separately below in the following order: first mode, second mode, second mode sub-harmonic.

First Mode In the frequency band, $65 \mathrm{kHz}$ to $85 \mathrm{kHz}$, the existence of the first mode is established from Figs. 13-19. The first mode remains unstable throughout the entire streamwise range surveyed but the amplification rate remains below 0.005 . These observations compare well with LST ${ }^{10}$. Since the oblique first mode disturbances are most unstable in supersonic flows ${ }^{16}$, only a component of the first mode waves are measured using the present single hot-wire configuration. Thus, the $65-85 \mathrm{kHz}$ band is lower than the actual first mode frequency range, but the degree of frequency shift cannot be determined from the present data.

Second Mode The frequency range, $\mathrm{f}=210 \mathrm{kHz}$ to 290 $\mathrm{kHz}$, is associated with the second mode. For $\mathrm{R}=1785$, the second mode is barely detectable near $\mathrm{f}=220 \mathrm{kHz}$. Further downstream, at $\mathrm{R}=1850$, the second mode becomes unstable $\left(-\alpha_{i}>0\right)$ in a small band around $f=225 \mathrm{kHz}$. At $\mathrm{R}=1945$, the second mode amplification rates increase substantially over a fairly large frequency band. The amplification rates increase at the next location, $\mathrm{R}=1975$, but decrease montonically in the downstream direction for $\mathrm{R}>1975$. However, the second mode remains unstable to the last measurement location, $R=2120$. As seen clearly in Figs. 15-18, the frequency band associated with the second mode maximum amplification rate and maximum amplitude do not coincide. This occurs since the unstable second mode amplitudes shift steadily to higher frequencies due to the overall thinning of the boundary layer in the downstream direction (i.e. boundary layer tuning). Thus, the maximum amplification rate is shifted to the higher frequency side of the unstable second mode frequency band. A similar observation was made by Stetson ${ }^{6}$.

Sub-harmonic The frequency band, 110-130 kHz, associated with the second mode sub-harmonic was not clearly identifiable from Fig. 12. However, the data of Fig. 12 were obtained using a hot-wire probe with a length-todiameter ratio that is approximately $25 \%$ lower than used for the Fig. 10 data and thus the sub-harmonic may have been attenuated. Note that unlike harmonics, which are associated with nonlinearities ${ }^{17}$ of the second mode, the sub-harmonic is considered to be a separate mode of oscillation similar to the secondary, sub-harmonic, helical disturbances used as forcing frequencies in previous $\mathrm{PSE}^{18}$ and $\mathrm{DNS}^{19}$ studies. For the range, $\mathrm{R}=1785-1945$, the $\mathrm{S} / \mathrm{N} \approx 1$ in the sub-harmonic frequency band. At $\mathrm{R}=1945$, the $\mathrm{S} / \mathrm{N}$ is slightly greater than 1 , and shows the most upstream detection of the sub-harmonic. Slightly downstream, $R=1975$, the sub-harmonic first becomes unstable. In contrast, initial instability of the second mode occurs at $\mathrm{R}=1850$. Furthermore, $\mathrm{R}=1975$ marks the location of a rapid rise in amplification rates for the subharmonic. In contrast, the second mode rapid rise in amplification occurs at $\mathrm{R}=1945$. The sub-harmonic amplification rates increase for the next location, $\mathrm{R}=2005$, but decrease montonically for $\mathrm{R}>2005$. However, the sub-harmonic remains unstable up to the last measurement location, $\mathrm{R}=2120$. Similar trends are observed for $\mathrm{R} \geq$ 1975 for the second mode. Thus, the overall downstream character of the sub-harmonic is similar to the second mode. However, the maximum amplification rate is shifted downstream for the sub-harmonic relative to the second mode.

The PSE study of Ref. 18 indicates that mild secondary instability of the forced sub-harmonic helical mode occurs at $10 \%$ mass flux fluctuations. From Fig. 8, the mass flux is $10 \%$ or greater for the last 3 streamwise locations, $\mathrm{R}$ > 2060 , corresponding to the region of the sub-harmonic growth in Fig. 10. However, due to the obliquity of these disturbances, only a component of the sub-harmonic is measured in the present investigation. Thus, the possible obliquity of the second mode disturbances in the present experiment cannot be ascertained. As a result, previous PSE $^{18}$ and DNS 20 studies, which use a helical pair of second mode disturbances as forcing inputs may not be suited for direct comparison. Rather, PSE or DNS studies using a 2D second mode as forcing, or additional experimental measurements, are needed for direct comparisons. 
In the following figures, 20-21, the amplification rates as a function of $\mathrm{R}$ are presented. Since the "noise" was not subtracted from the full CVA output voltages, the amplitudes over the upstream regions, $\mathrm{R}<1910$, approach the noise level of the CVA. Thus, in this region, the amplification rates are zero (i.e. the derivative of a constant is zero). However, these upstream regions, $\mathrm{R}<$ 1910, do not represent points on a neutral stability diagram. Only the region, $\mathrm{R}>1910$, should be considered representative of the "true" growth of the flow.

Several amplification rate curves are presented for a few frequencies in Fig. 20. The selective amplification of particular frequencies as the boundary layer thickness decreases in the downstream direction is shown. The frequency, $\mathrm{f}=80 \mathrm{kHz}$, is within the frequency band associated with the first mode and, $\mathrm{f}=254 \mathrm{kHz}$, is within the frequency band associated with the second mode. In the linear stability region, $\mathrm{R}=2020$, the $80 \mathrm{kHz}$ experimental dimensional amplification rate is approximately $4.34 / \mathrm{ft}$, which is within $11 \%$ of the $\operatorname{LST}^{10}$ result of $4.85 / \mathrm{ft}$. At the location of maximum LST second mode amplification rate, $\mathrm{R}=2020$, the LST dimensional amplification rate is approximately $13.88 / \mathrm{ft}$ for $\mathrm{f}=230 \mathrm{kHz}$ which compares reasonably well (within $7 \%$ ) to the experimental dimensional amplification rate of $12.89 / \mathrm{ft}$ for $\mathrm{f}=234 \mathrm{kHz}$. Furthermore, for $\mathrm{R} \leq 2120$, the location of maximum amplification rate for the most unstable frequency (i.e. maximum $\mathrm{N}$-factor) occurs at $\mathrm{R} \approx 1975$ for both experiment $(\mathrm{f}=254 \mathrm{kHz})$ and $\operatorname{LST}(\mathrm{f}=220 \mathrm{kHz})$. Overall, these comparisons are relatively good considering the possible effects of model misalignment.

The maximum amplification rates associated with the first mode, sub-harmonic of the second mode, and second mode are presented in Fig. 21. The maximum amplification rates were calculated by determining the maximum growth rate over the $65-85 \mathrm{kHz}$ (first mode), $210-290 \mathrm{kHz}$ (second mode), and 110-130 kHz (subharmonic) ranges for each R-location. Over the upstream region, $\mathrm{R} \leq 1910$, the $\mathrm{S} / \mathrm{N} \approx 1$ and therefore the amplification rates are unreliable. Further downstream, the sub-harmonic growth is similar in character to the second mode, but the maximum amplification rate is shifted downstream by about $\Delta \mathrm{R}=30$. However, the sub-harmonic growth rate is not explosive and is on the order of the second mode growth rate, also comparing well with $\mathrm{PSE}^{18}$. Furthermore, the transition onset, $\mathrm{R}=1960-1990$, occurs in a region where the second mode is dominant. Thus, transition is not caused by the sub-harmonic secondary instability; this is also observed from PSE ${ }^{18}$ theory. However, relatively "far" downstream of transition onset, $\mathrm{R}>2060$, all the amplification rates are comparable. (Note: in the downstream region, $\mathrm{R} \geq 1975$, the "first mode growth" partially reflects the lowfrequency growth and is not necessarily characteristic of the first mode itself).

The wavelengths for the second mode and second mode sub-harmonic, normalized with respect to the boundary layer thickness, are presented in Fig. 22. The error in $\lambda / \delta$ is $4 \%$ of the plotted values. The disturbance wavelengths were estimated assuming the phase velocity is $90 \%$ the boundary layer edge velocity predicted from computations. ${ }^{14}$ Upstream, $1785 \leq \mathrm{R} \leq 1945$, the second mode wavelength scales as: $\lambda \approx 2.2 \delta$. Further downstream in the transitional region, $1975 \leq \mathrm{R} \leq 2120$, the second mode wavelength scales as: $\lambda \approx 2 \delta$. For the transitional region, the $\lambda \approx 2 \delta$ scaling compares well with sharp straight cone data ${ }^{6}$. Over the range, $1975 \leq \mathrm{R} \leq 2120$, the sub-harmonic scales as, $\lambda \approx 4.3 \delta$. This is expected since the sub-harmonic frequencies are approximately half the second mode. Since the sub-harmonic is oblique, only a component of the disturbance is measured and the measured frequency is lower than the actual frequency. Furthermore, the actual phase velocity for the oblique wave is unknown. Thus, the $\lambda \approx 4.3 \delta$ scaling should be considered approximate.

\section{Fluctuation Data - Effect of Model Misalignment}

The boundary layer thickness distribution, Fig. 8, and fluctuation spectra, Fig. 10, suggested that there may be a misalignment of the model. The misalignment of the boundary layer measurement ray was estimated at $0.2^{\circ}$ windward. An additional test was therefore conducted with the model boundary layer measurement ray aligned as carefully as possible to zero degrees yaw. Subsequent analysis showed that these additional measurements were conducted on a measurement ray less than $0.1^{\circ}$ to the leeward side. Furthermore, these additional measurements were conducted with an improved CVA system that had a $\mathrm{S} / \mathrm{N}$ ratio approximately an order of magnitude greater than the previous system. (Note that these data were obtained using a low-pass cut-off frequency of $1 \mathrm{MHz}$ ). The results from this additional test are presented below.

The fluctuation spectra at $\mathrm{R}=2120$ are shown in Fig. 23 . At this streamwise location, the maximum amplitudes for the second mode and first harmonic occur at $226 \mathrm{kHz}$ and $449 \mathrm{kHz}$, respectively. Thus, the first harmonic frequency is approximately twice the fundamental as expected. A first harmonic was also observed for the more windward case by operating the CVA at maximum, practicable $\mathrm{S} / \mathrm{N}$ conditions. Furthermore, a second harmonic of the fundamental is also shown in the figure. The maximum amplitude of the second harmonic occurs at $670 \mathrm{kHz}$ which is approximately a factor of 3 larger than the fundamental, $\mathrm{f}=226 \mathrm{kHz}$, as expected. Overall, the nonlinear region extends over a larger region for this case relative to the more windward case which is consistent with the downstream movement of transition along the windward side of the model.

"N-factor" The "N-factor", or integrated growth rates, are presented at select frequencies in Fig. 24. (To compare LST with the experimental data, the LST N-factor, 6.015, at the most upstream measurement location was added to the experimental integrated growth rates). The positive, constant slope line regions mark the linear stability regime, or exponential growth region. For the frequencies, $215-230 \mathrm{kHz}$, the linear stability regime spans the range, $1880 \leq \mathrm{R} \leq 2060$. Over this streamwise range, the change in $\mathrm{N}$-factor is 3.05 and 2.94 for $\mathrm{f}=219 \mathrm{kHz}$ and $\mathrm{f}=230 \mathrm{kHz}$, respectively. This compares remarkably well with LST. ${ }^{10}$ For $\mathrm{f}=220 \mathrm{kHz}$, the change in LST N-factor is 3.10 for the range $1880 \leq \mathrm{R} \leq 2060$, comparing within $1.5 \%$ of the experimental data. The slope for this frequency also 
compares well with LST which is presented as the solid line in the figure. For $\mathrm{f}=230 \mathrm{kHz}$, the change in LST integrated growth rate is 3.09 for the range $1880 \leq \mathrm{R} \leq$ 2060 , comparing within $5 \%$ of the experimental data. In summary, these results suggest that excellent comparison in terms of integrated growth rates are obtained in a quiet wind tunnel in the linear stability regime.

\section{Concluding Remarks}

The first hypersonic boundary layer stability measurements in a quiet tunnel have been obtained. All test cases were conducted at a freestream Mach number of 5.91 and freestream unit Reynolds number of $2.82 \times 10^{6} / \mathrm{ft}$ in the NASA Langley Research Center's Nozzle Test Chamber Facility. The significant conclusions are summarized as follows:

1. The second mode is the most unstable disturbance mode. This mode is responsible for transition onset which is estimated in the range, $\mathrm{R}=1960-1990$.

2 . The second mode integrated growth rates compare within $1.5-5 \%$ of linear stability theory in the linear stability regime.

3. The second mode wavelength is approximately twice the boundary layer thickness verifying the boundary layer tuning observed in conventional tunnels.

4. In the vicinity of maximum amplification of the second mode, the first mode amplification rates are within $11 \%$ of linear stability theory.

5. The second mode sub-harmonic rapid disturbance growth region occurs at $10 \%$ mass flux fluctuations, comparing well with parabolized stability equation theory. The sub-harmonic wavelength scaling is about a factor of 4.3 greater than the boundary layer thickness in the transitional region.

6. Both first and second harmonics of the second mode exist downstream of the rapid disturbance second mode growth region. Thus, these non-linearities are not attributed to "high" freestream disturbance levels.

\section{Acknowledgments}

This work was supported by a cooperative agreement between North Carolina State University and NASA LaRC under the Cooperative Agreement NCC1-183. The authors thank A. E. Blanchard for making the hot-wire probes. The CVA system used in this work was developed by Tao Systems under SBIR Contract No. NAS1-20192. The authors are grateful to G.R. Sarma for providing assistance with the CVA.

\section{$\underline{\text { References }}$}

${ }^{1}$ Finley, D. "Hypersonic Aerodynamic Considerations and Challenges," AIAA 90-5222, Oct. 1990.

${ }^{2}$ Stetson, K.F. and Kimmel, R.L., "On Hypersonic Boundary-Layer Stability,” AIAA 92-0737, Jan. 1992.

${ }^{3}$ Laufer, J., "Aerodynamic Noise in Supersonic Wind Tunnels," J. Aerospace Sciences, Vol. 28, No. 9, Sept. 1961, pp. 685-692.

${ }^{4}$ Morkovin, M.V., "On Supersonic Wind Tunnels with Low Free-Stream Disturbances," Journal of Applied

Mechanics, Vol. 26, Sept. 1959, pp. 319-324.
${ }^{5}$ Mack, L.M., "Stability of Axisymmetric Boundary Layers on Sharp Cones at Hypersonic Mach Numbers," AIAA 87-1413, June 1987.

${ }^{6}$ Stetson, K.F., Thompson, E.R., Donaldson, J.C., and Siler, L.G., "Laminar Boundary Layer Stability Experiments on a Cone at Mach 8, Part 1: Sharp Cone," AIAA 83-1761, July 1983.

${ }^{7}$ Wilkinson, S.P., Anders, S.G., Chen F.-J., and White J.A., "Status of NASA Langley Quiet Flow Facility Developments," AIAA 94-2498, June 1994.

${ }^{8}$ Blanchard, A.E., Lachowicz, J.T., and Wikinson, S.P., "Performance of the NASA-Langley Mach 6 Quiet Wind Tunnel," AIAA 96-0441, Jan. 1996.

${ }^{9}$ Chen, F.-J., Wilkinson, S.P., and Beckwidth, I.E., "Görtler Instability and Hypersonic Quiet Nozzle Design," Journal of Spacecraft and Rockets, Vol. 30, No. 2, MarchApril 1993, pp. 170-175.

${ }^{10}$ Balakumar, P. and Malik, M.R., "Effects of Adverse Pressure Gradient and Wall Cooling on Instability of Hypersonic Boundary Layers," HTC-9404, March 1994.

${ }^{11}$ Sarma, G.R., "Analysis of a Constant Voltage Anemometer Circuit," IEEE/IMTC Conference, Irvine, CA, May 18-20, 1993.

${ }^{12}$ Lachowicz, J.T., "Hypersonic Boundary Layer Stability Experiments in a Quiet Wind Tunnel with Bluntness Effects," Ph.D. Dissertation, Mechanical and Aerospace Engineering Dept., North Carolina State University, Raleigh, NC, Nov. 1995.

13 Kendall, J.M., "Some Comparisons of Linear Stability Theory with Experiment at Supersonic and Hypersonic Speed," Instability and Transition, M.Y. Hussaini and R.G. Voigt, Eds., Vol. 1, Springer-Verlag, NY, 1989, pp. 68-76.

${ }^{14}$ Edwards, J.R., "A Low-Diffusion Flux-Splitting Scheme for Navier-Stokes Calculations," AIAA 95-1703, June 1995.

${ }^{15}$ Kimmel, R., Demetriades, A., and Donaldson, J., "Space-Time Correlation Measurements in a Hypersonic Transitional Boundary Layer,” AIAA 95-2292, June 1995.

16Mack, L.M., "Boundary Layer Stability Theory," Jet Propulsion Lab. Report No. 900-277 Rev. A, Pasadena, Ca., Nov. 1969.

${ }^{17}$ Kimmel, R.L., and Kendall, J.M., "Nonlinear Disturbances in a Hypersonic Laminar Boundary Layer," AIAA 91-0320, Jan. 1991.

${ }^{18}$ Chang, C.-L., and Malik, M.R., "Non-Parallel Stability of Compressible Boundary Layers," AIAA 932912, July 1993.

${ }^{19}$ Pruett, C.D., "Direct Numerical Simulation of Laminar Breakdown in High-Speed, Axisymmetric Boundary Layers," AIAA 92-0742, Jan. 1992.

${ }^{20}$ Pruett, C.D., and Chang C.-L., "Spatial Direct Numerical Simulation of High-Speed Boundary-Layer Flows, Part II: Transition on Cone in Mach 8 Flow," 
Theoretical and Computational Fluid Dynamics, To Appear.

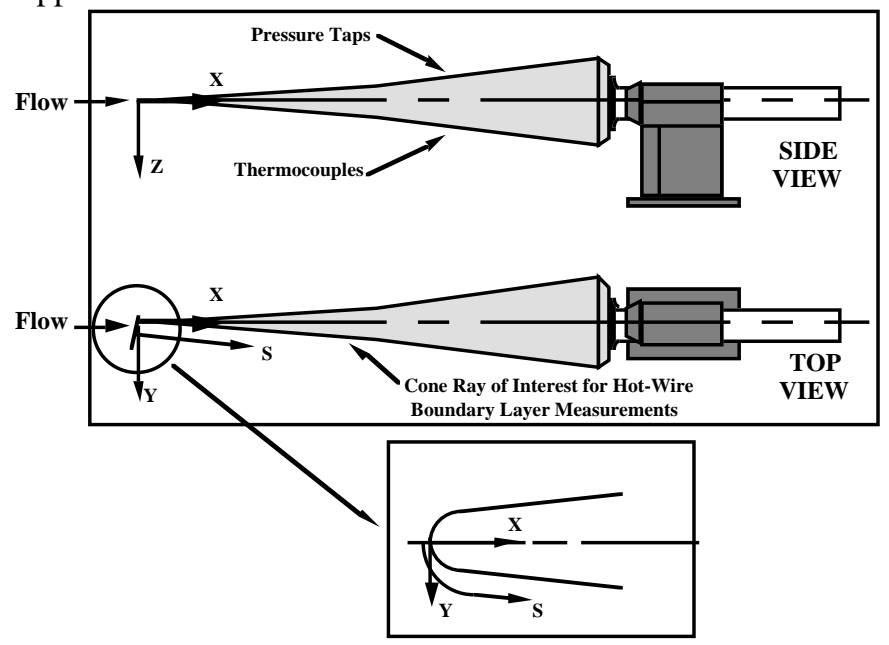

Fig. 1 Flared-Cone Coordinate System.

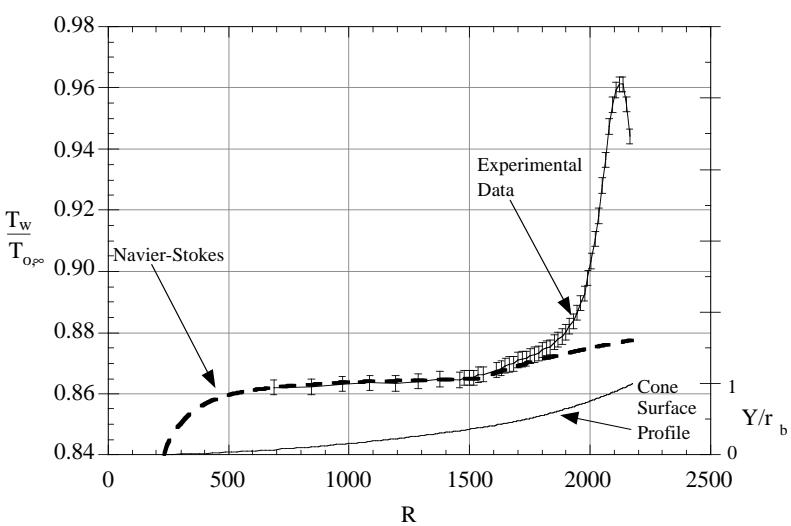

Fig. 2 Surface Temperature Distribution.

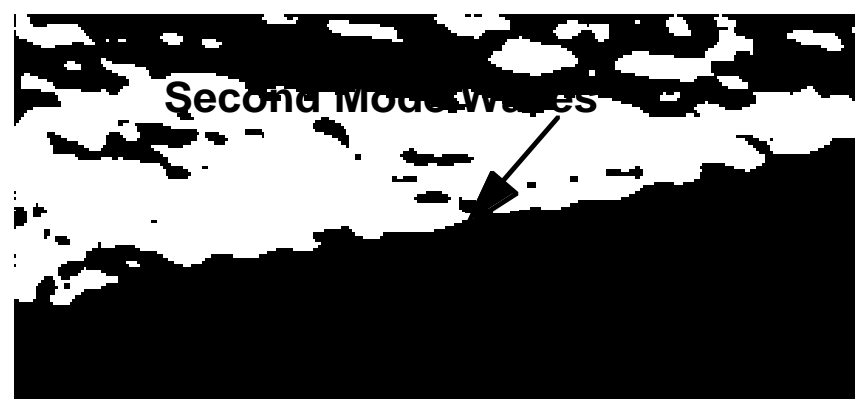

Fig. 3 Schlieren Photograph over aft region of Model.

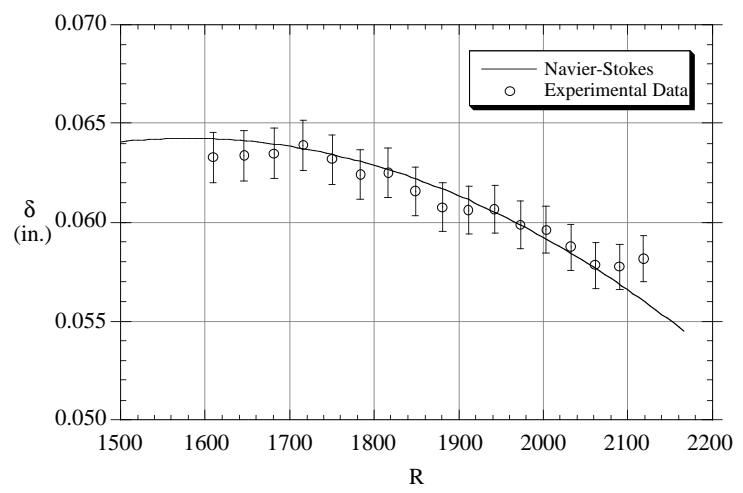

Fig. 4 Boundary Layer Thickness Distribution.

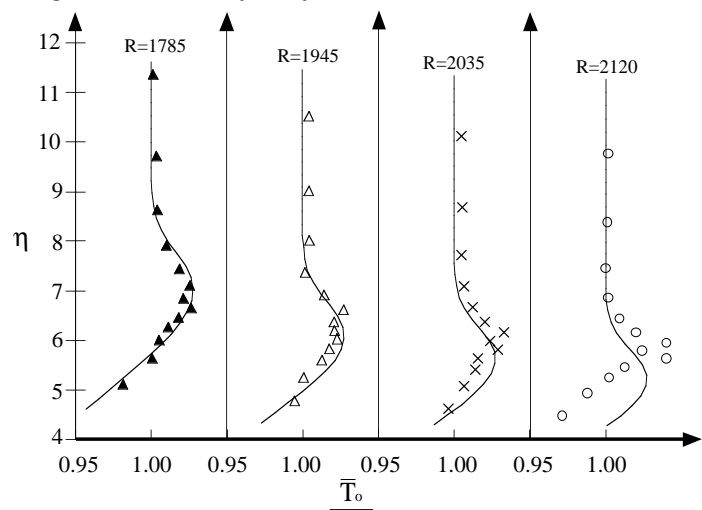

$\overline{\left(\overline{\bar{T}_{0}}\right)_{\infty}}$

Fig. 5 Total Temperature Profiles.

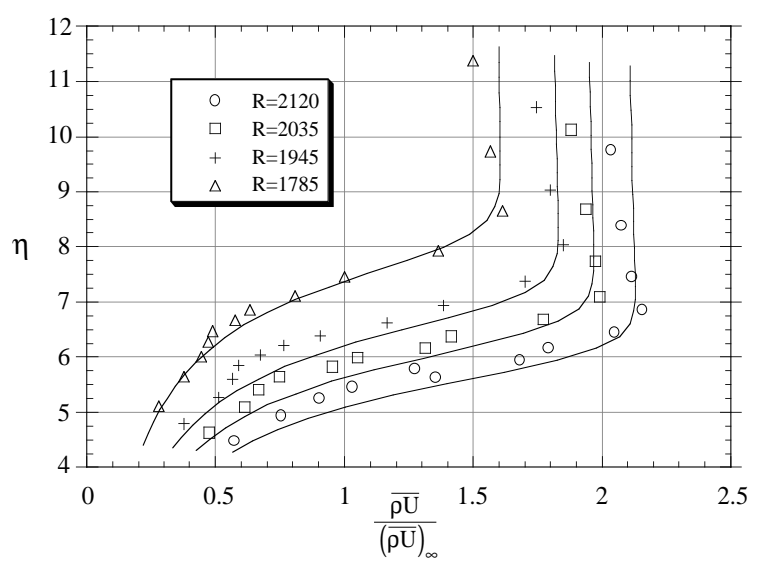

Fig. 6 Mass Flux Profiles.

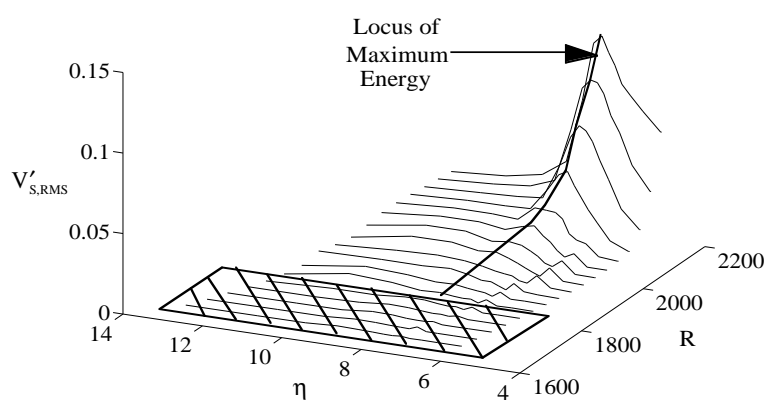

Fig. 7 Uncalibrated RMS Profiles. 


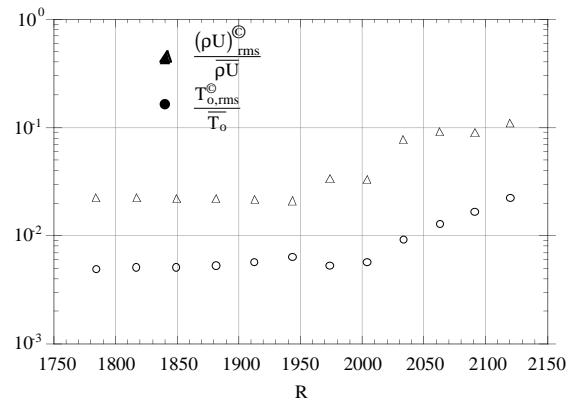

Fig. 8 Streamwise RMS Mass Flux and Total Temperatures.

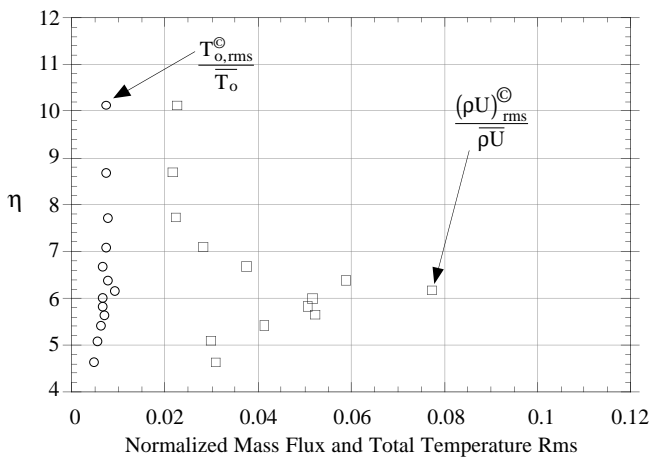

Fig. 9 RMS Mass Flux and Total Temperature Profiles at $\mathrm{R}=2035$.

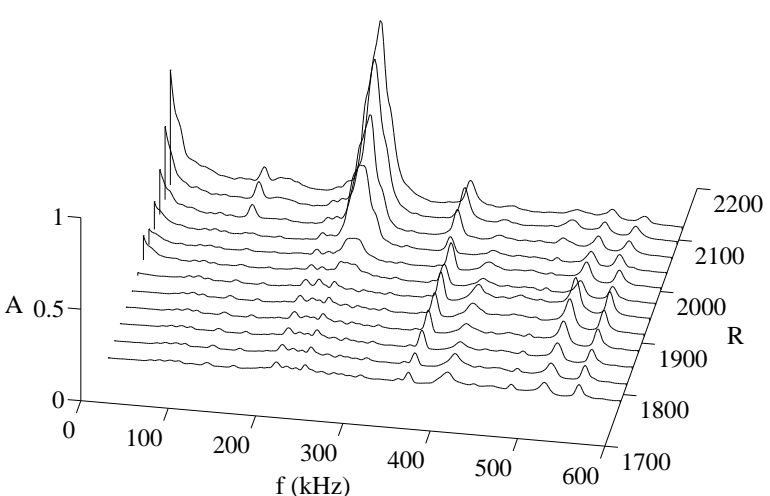

Fig. 10 Fluctuation Spectra at Maximum Energy Locations.

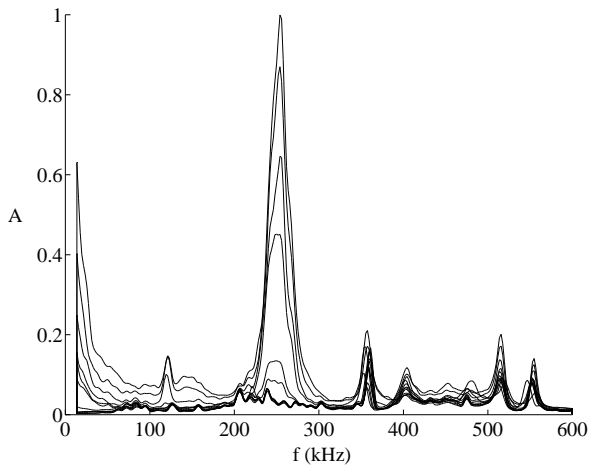

Fig. 11 Frontal View of Fluctuation Spectra at Maximum Energy Locations.

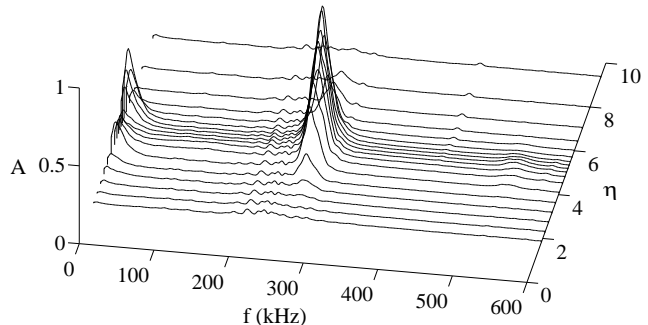

Fig. 12 Spectra through the boundary layer at $\mathrm{R}=2120$.

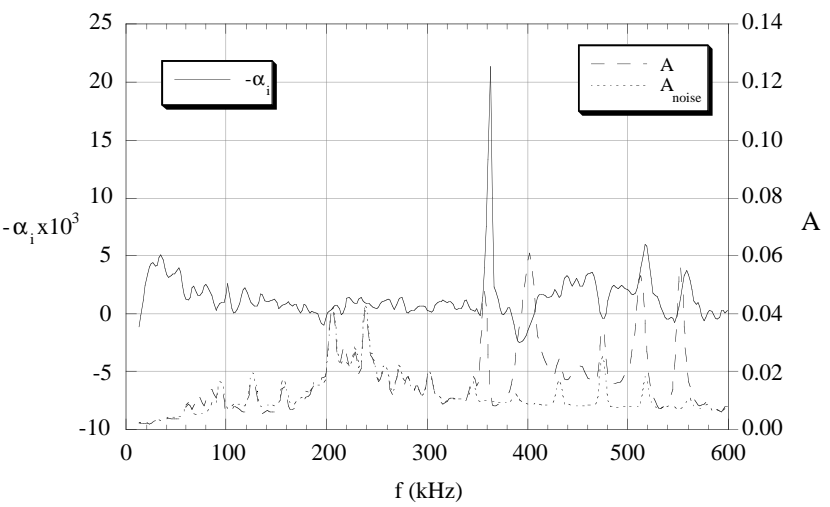

Fig. 13 Amplitudes and amplification rates at $R=1785$.

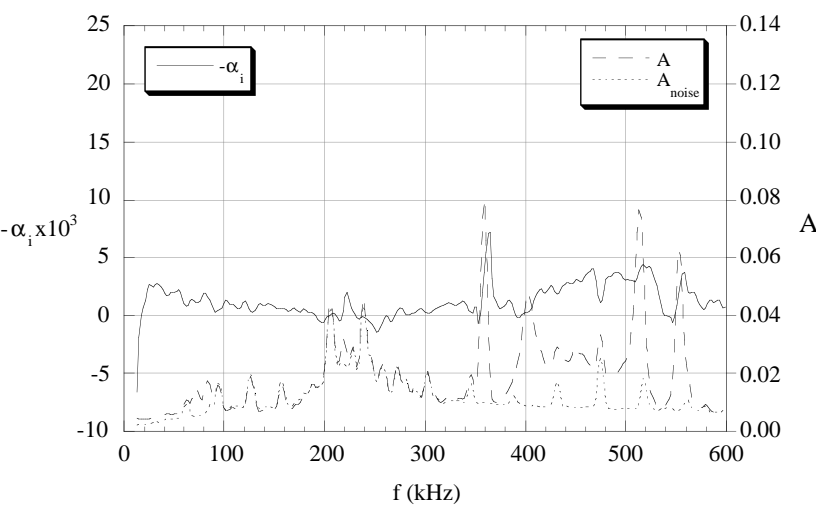

Fig. 14 Amplitudes and amplification rates at $R=1850$.

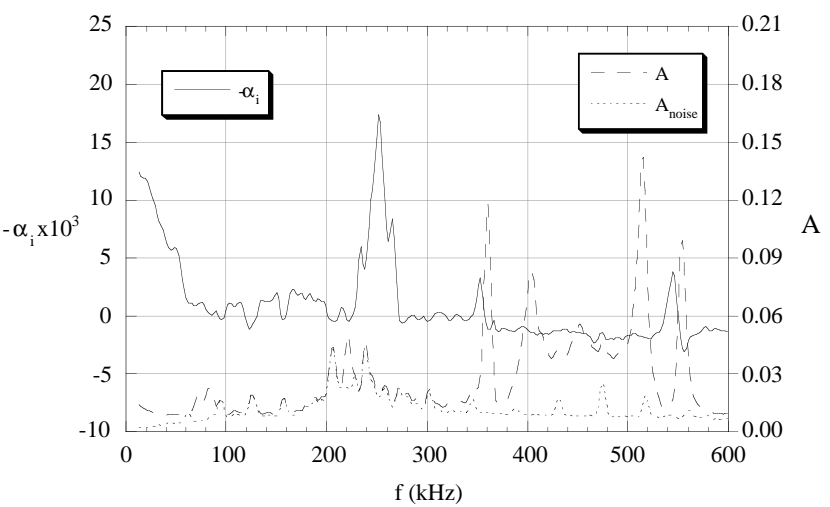

Fig. 15 Amplitudes and amplification rates at $\mathrm{R}=1945$. 


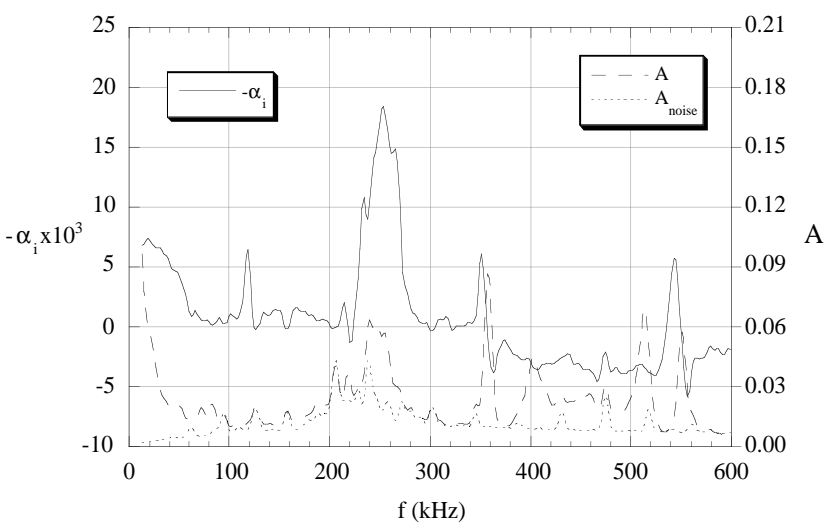

Fig. 16 Amplitudes and amplification rates at $\mathrm{R}=1975$.

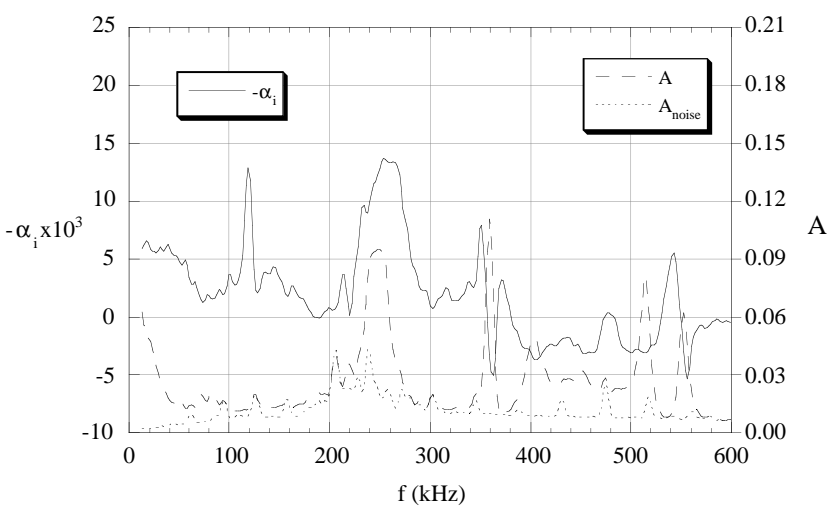

Fig. 17 Amplitudes and amplification rates at $\mathrm{R}=2005$.

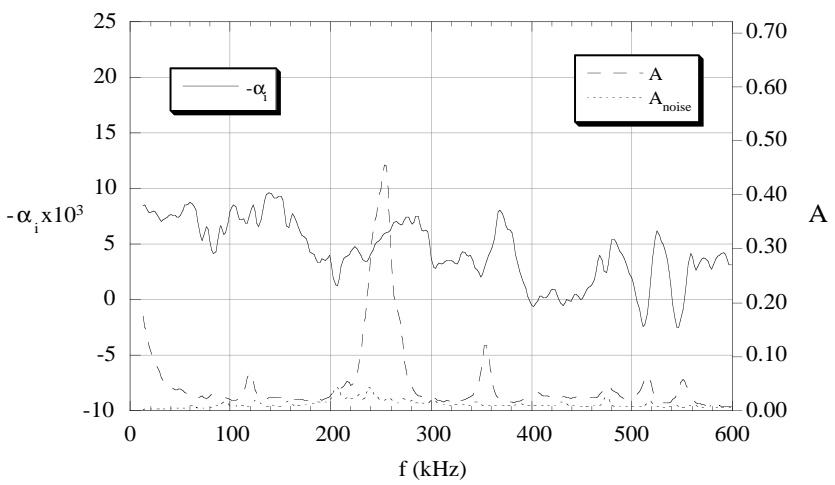

Fig. 18 Amplitudes and amplification rates at $R=2065$.

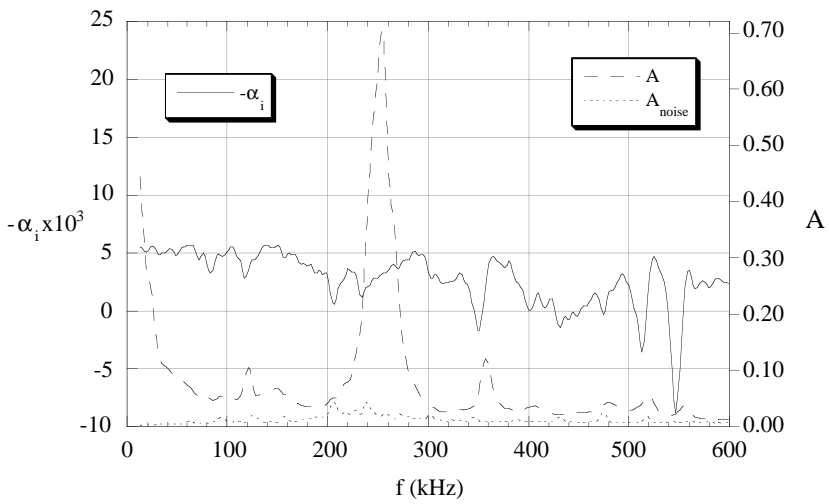

Fig. 19 Amplitudes and amplification rates at $\mathrm{R}=2120$.

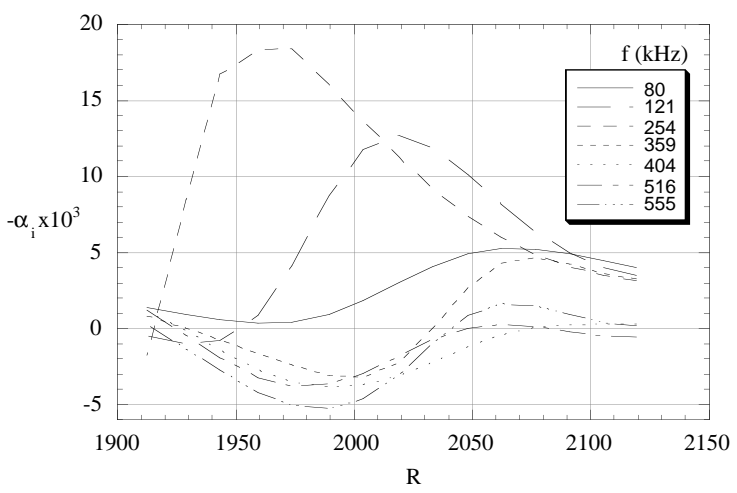

Fig. 20 Amplification rates vs. $\mathrm{R}$ at select frequencies.

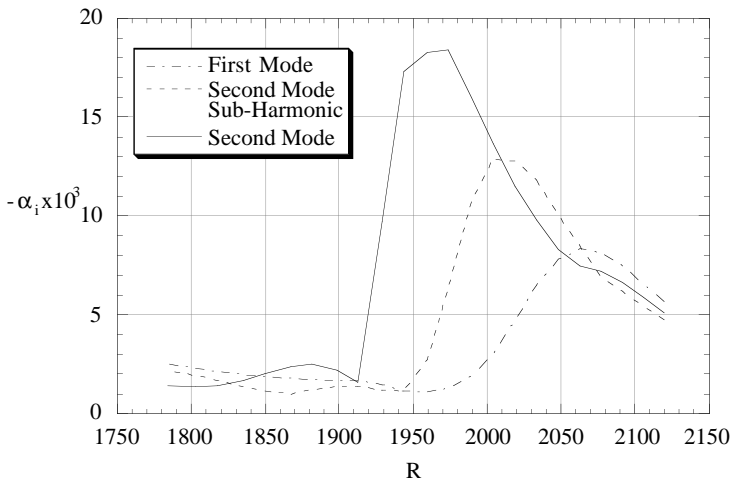

Fig. 21 Maximum amplification rates of the first \& second modes, and the second mode subharmonic.

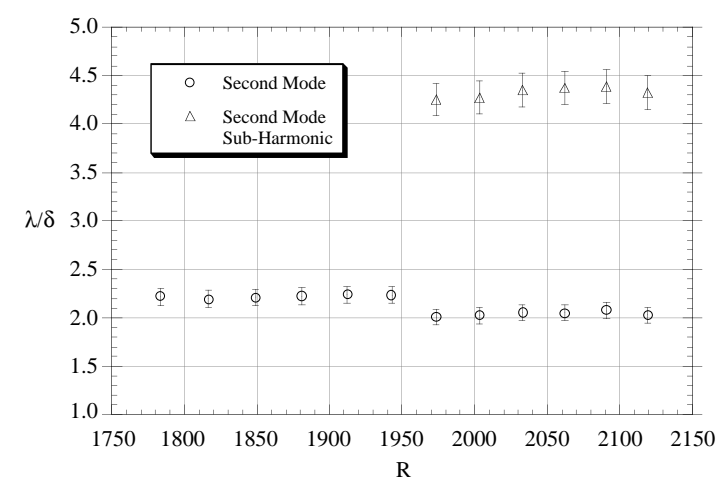

Fig. 22 Second Mode Fluctuation Wavelength.

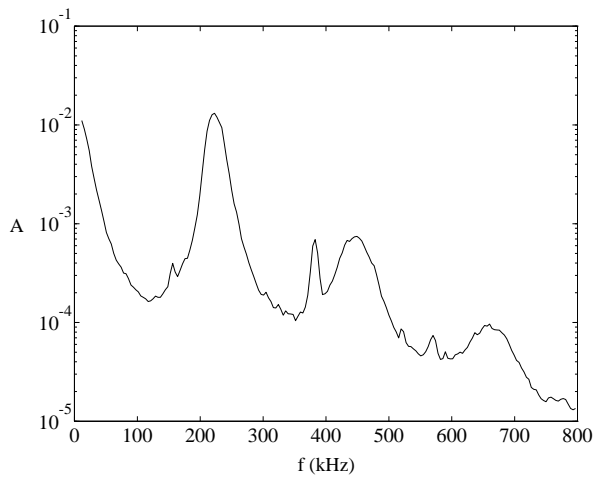

Fig. 23 Fluctuation Spectra at $R=2120$. 


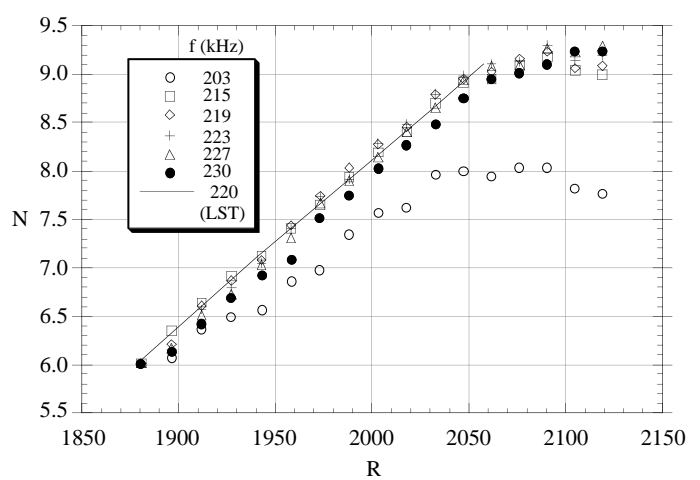

Fig. 24 Second Mode "N-factors" at Select Frequencies. 